\title{
Nomenclature for kidney function and disease: executive summary from a KDIGO consensus conference
}

\author{
Andrew S. Levey ${ }^{1 凶}{ }^{\boxplus}$, Kai-Uwe Eckardt ${ }^{2 凶}$, Nijsje M. Dorman $\mathbb{1}^{3}$, Stacy L. Christiansen ${ }^{4}$, \\ Michael Cheung 5 , Michel Jadoul ${ }^{6}$ and Wolfgang C. Winkelmayer ${ }^{7}$ \\ An obligation of medical journals is the responsible, professional and expeditious delivery of \\ knowledge from researchers and practitioners to the community. The task of editors, therefore, \\ rests not merely in selecting what to publish, but in judging how it might best be communicated. \\ The challenge of improving descriptions of kidney function and disease in medical publishing \\ was the impetus for a KDIGO consensus conference. The conference goals included standardizing \\ and refining kidney-related nomenclature and developing a glossary to be used by journals.
}

'Division of Nephrology, Tufts Medical Center, Boston, MA, USA.

${ }^{2}$ Department of Nephrology and Medical Intensive Care, Charité-Universitätsmedizin Berlin, Berlin, Germany.

${ }^{3}$ Managing Editor, American Journal of Kidney Diseases, University of Pennsylvania, Philadelphia, PA, USA.

${ }^{4}$ Managing Editor, The Journal of the American Medical Association, JAMA Network, Chicago, IL, USA. ${ }^{5} \mathrm{KDICO}$, Brussels, Belgium. ${ }^{6}$ Cliniques Universitaires Saint Luc, Université Catholique de Louvain, Brussels, Belgium.

${ }^{7}$ Selzman Institute for Kidney Health, Section of Nephrology, Department of Medicine, Baylor College of Medicine, Houston, TX, USA.

凶e-mail: alevey@

tuftsmedicalcenter.org;

kai-uwe.eckardt@charite.de

https://doi.org/10.1038/

s41581-020-0290-9
The rationale for the Kidney Disease: Improving Global Outcomes (KDIGO) Consensus Conference held in June 2019 (REF. ${ }^{1}$ ) is that the worldwide burden of kidney disease is rising but public awareness remains limited, underscoring the need for effective communication by stakeholders in the kidney health community ${ }^{2-5}$. Despite this need, the nomenclature for describing kidney function and disease lacks uniformity and clarity. Two decades ago, a survey of published articles and meeting abstracts reported an array of overlapping, confusing terms for chronic kidney disease (CKD) and advocated adoption of unambiguous terminology $\mathrm{y}^{6}$. Nevertheless, terms flagged by that analysis as being problematic still appear in publications. A coherent, shared nomenclature could improve communication, not only to better appreciate the burden of disease but also to aid understanding of how patients feel about their disease, enable more effective communication between kidney disease specialists and other clinicians, enable more straightforward comparison and integration of datasets, improve recognition of knowledge gaps and facilitate more comprehensive public health policies for acute and chronic kidney disease.

Developing consistent, patient-centred and precise descriptions of kidney function and disease in the scientific literature is important to align communication in clinical practice, research and public health. The goal is to facilitate communication within and across disciplines and between practitioners and patients, with the ultimate hope of improving outcomes through consistency and precision.

Attendees at the conference included editors of kidney subspecialty journals, kidney subspecialty editors at general medical journals and journals from other subspecialties, experienced authors of clinical kidney health research, and patients. Guiding principles of the conference were that the revised nomenclature should be patient-centred, precise and consistent with nomenclature used in the KDIGO guidelines. The discussion focused on general descriptions of acute and chronic kidney disease and kidney measures, rather than specific kidney diseases and particular measures of function and structure.

As described in the conference report ${ }^{7}$, the meeting attendees reached general consensus on the following recommendations. First, to use 'kidney' rather than 'renal' or 'nephro-' when referring to kidney disease and kidney function; second, to use 'kidney failure' with appropriate descriptions of presence or absence of symptoms, signs and treatment rather than 'end-stage' kidney disease; third, to use the KDIGO definition and classification of acute kidney diseases and disorders (AKD) and acute kidney injury (AKI) rather than alternative descriptions to define and classify severity of AKD and AKI; fourth, to use the KDIGO definition and classification of CKD rather than alternative descriptions to define and classify CKD; and fifth, to use specific kidney measures, such as albuminuria or decreased glomerular filtration rate, rather than 'abnormal' or 'reduced' kidney function to describe alterations in kidney structure and function (Supplementary Box 1). The proposed glossary contains five corresponding sections and comprises specific items for which there was general agreement among the conference participants (Supplementary Table 1) 7 . For each section, the glossary includes preferred terms, abbreviations, descriptions, and terms to avoid, with acknowledgment that journals may choose which of the recommendations to implement, and that journal style will dictate when and how to abbreviate terms. 
A guiding principle for the development of the glossary was patient-centredness. The Health and Medicine Division of the US National Academies of Sciences defines patient-centred care as "Providing care that is respectful of, and responsive to, individual patient preferences, needs and values, and ensuring that patient

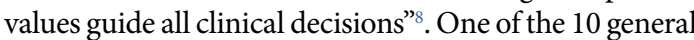
principles recommended for redesign of the health system is that "Knowledge is shared and information flows freely. Patients should have unfettered access to their own medical information and to clinical knowledge. Clinicians and patients should communicate effectively and share information." In principle, the terms used to describe kidney function and disease should be understandable to all, with acknowledgement of variation in the level of health literacy. Use of multiple terms with similar meaning can lead to confusion, as can use of terms that forecast the future (such as 'pre-dialysis'). However, convergence of multiple names into an accepted set of terms requires that users of the glossary are willing to accept that labels that were pre-eminent historically should now be superseded.

Of equal importance to patient-centredness in the development of the glossary was precision. It is anticipated that refinements that result in more precise disease descriptions will be incorporated into current nomenclature for kidney function and disease, rather than replace it altogether. Thus, although the glossary is designed to be consistent with current knowledge and is stable enough to remain relevant for the foreseeable future, it is also intended to be sufficiently flexible to accommodate new vocabulary arising with advances in the field.

A central strength of the proposed glossary is that it is based on existing KDIGO definitions, classifications and nomenclature for acute and chronic kidney disease. In addition, it was developed using a systematic process, including articulation of a clear and transparent rationale (patient-centredness and precision); capture of stakeholder viewpoints via patient focus groups and a corresponding survey; a period of public comment on conference scope; and attainment of consensus among attendees at the conference. While the recommendations are not likely to answer all concerns, the consensus among conference attendees was that standardizing scientific nomenclature is a necessary first step to improving communication between clinicians, researchers and public health officials, and with patients, their families and caregivers, and the public.

Limitations of the proposed glossary are that it is restricted to English; only a limited number of stakeholders could participate for practical reasons; it is not comprehensive (it does not include disease classification, dialysis or transplantation); and further specification will be required for studies in children. For these and other reasons, we consider the current recommendations for a glossary as an important starting point, and it will require future expansion and updating.

Achieving consensus among conference attendees and publication of the conference report and glossary are only the first steps in implementation of a revised nomenclature. The glossary will be freely available on the KDIGO website. Elements of the glossary will be included in online updates to the newly released (11th) edition of the AMA Manual of Style. Medical journals adopting the recommendations will need to determine how to implement them and this process will require education of editorial staff as well as proactive communication with authors. If successful, further implementation in clinical practice, research and public health will require widespread dissemination and professional education. Improving communication with patients and the public will require efforts to improve patient education and health literacy for the public and guides to communication with patients. Professional societies, industry and patient advocacy organizations will be crucial to these efforts.

Advances in research, particularly in precision medicine, will introduce a myriad of new terms and novel concepts requiring incorporation into disease definitions and classifications. In addition, the increasing prominence and participation of patient and caregiver communities in defining research objectives and best practices in clinical care will further elucidate the characteristics of patient-centred terminology. Expanding and updating the KDIGO glossary can be accomplished as part of the activities of future KDIGO guideline workgroups and conferences.

1. Kidney Disease: International Global Outcomes. Consensus Conference on Nomenclature for Kidney Function \& Disease. https://kdigo.org/conferences/nomenclature/ (2019)

2. Plantinga, L. C. et al. Arch. Intern. Med. 168, 2268-2275 (2008).

3. James, S. L. Lancet 392, 1789-1858 (2018).

4. GBD Chronic Kidney Disease Collaboration. Lancet 395, 709-733 (2020).

5. Saran, R. et al. Am. J. Kidney Dis. 71 (3 Suppl. 1), S1-S676 (2018).

6. Hsu, C. Y. \& Chertow, G. M. Am. J. Kidney Dis. 36, 415-418 (2000).

7. Levey, A. et al. Kidney Int. https://doi.org/10.1016/j.kint.2020. 02.010 (2020)

8. Institute of Medicine Committee on Quality of Healthcare in America. Crossing the Quality Chasm: A New Health System $f$ or the 21st Century (National Academies Press, 2001).

Acknowledgements

The authors are grateful to Juhi Chaudhari, Tufts Medical Center, Boston for assistance with manuscript preparation before submission. The conference was sponsored by KDICO and was in part supported by unrestricted educational grants from AstraZeneca, Bayer HealthCare, Boehringer Ingelheim, Fresenius Medical Care, Roche and Sanofi. The content of this article does not necessarily reflect the views or opinions of the organizations or journals that were represented at the conference. Responsibility for the information and views expressed is limited to the coauthors. Note that this Comment article is being published concurrently in multiple journals and serves as an executive summary of the full report published in Kidney International (Levey, A. et al. Kidney Int. https://doi.org/10.1016/ j.kint.2020.02.010 (2020)). These articles cover identical concepts but vary in minor stylistic and spelling changes, detail and length of manuscript, in keeping with each journal's style. Excerpts are adapted with permission of KDIGO and the International Society of Nephrology.

\section{Competing interests}

A.S.L. has received research support from AstraZeneca, NIDDK and NKF. K.U.E. has received consultancy fees from Akebia, Bayer and Genzyme; speaker honoraria from Bayer and Vifor; and research support from Amgen, AstraZeneca, Bayer, Fresenius Medical Care and Genzyme. N.M.D. has equity ownership/stock options from Eli Lilly $\&$ Co. M.J. has received consultancy fees from Astellas, AstraZeneca, GlaxoSmithKline, MSD and Vifor Fresenius Medical Care Renal Pharma; speaker honoraria from Abbvie, Amgen, Menarini, MSD and Vifor Fresenius Medical Care Renal Pharma; travel support from Amgen; and research support from Alexion, Amgen, Janssen-Cilag, Otsuka and Roche. W.C.W. has received consultancy fees from Akebia, AMAG, Amgen, AstraZeneca, Bayer, Daiichi-Sankyo, Relypsa and ZS Pharma; speaker honoraria from FibroGen; and research support from $\mathrm{NIH}$. The other authors declare no competing interests.

\section{Supplementary information}

Supplementary information is available for this paper at https://doi.org/ 10.1038/s41581-020-0290-9. 